\title{
Load-Aware On-Demand Routing (LAOR) Protocol for Mobile Ad hoc Networks
}

\author{
Joo-Han Song, Vincent Wong, and Victor C.M. Leung \\ Department of Electrical and Computer Engineering \\ The University of British Columbia \\ Vancouver, BC, Canada V6T 1Z4 \\ e-mail: \{joohans, vincentw, vleung\}@ece.ubc.ca
}

\begin{abstract}
Most current routing protocols for mobile ad hoc networks consider the shortest path with minimum hop counts as the optimal route. However, the minimum end-to-end delay from source to destination may not always be achieved through this shortest path. In this paper, we propose an efficient Delay-based Load-Aware On-demand Routing (D-LAOR) protocol, which determines the optimal path based on the estimated total path delay and the hop count. We demonstrate the effectiveness of DLAOR by integrating it with the Ad hoc On-demand Distance Vector (AODV) routing protocol. Simulation results, obtained using the ns-2 network simulation platform, show that D-LAOR scheme increases packet delivery fraction and decreases end-toend delay by more than $10 \%$ in a moderate network scenario when compared with the original AODV and other LAOR protocols.
\end{abstract}

\section{INTRODUCTION}

A Mobile Ad hoc NETwork (MANET) [1] consists of a set of wireless mobile nodes communicating to each other without any centralized control or fixed network infrastructure. MANETs have been evolving to serve a growing number of applications that rely on multi-hop wireless infrastructures that can be deployed quickly. The potential applications include emergency disaster relief, battlefield situations, mine site operations, and wireless classrooms or meeting rooms in which participants wish to share information or to acquire data.

Since one of the most important issues in MANETs is to find an efficient and reliable route from any source to any destination, current research on MANETs mainly focuses on ad-hoc routing protocols. These protocols can be broadly divided into three types [2]: flat routing, hierarchical routing, and geographic position assisted routing. Flat routing protocols use a flat addressing scheme and are further divided into two categories: proactive and on-demand. Proactive routing protocols make use of periodic routing updates while on-demand routing protocols only maintain the routes that are currently in use. Since on-demand routing protocols react quickly to topology changes, they perform better than proactive routing protocols in general [3]. Hierarchical routing protocols require a scalable addressing system. Geographic position assisted routing assumes each mobile node to be equipped with the global positioning system.

Although there are many proposed routing protocols for MANETs, most of them consider the shortest-path with minimum hop count as the route selection criterion. Even though this hop metric is easy to implement and reliable in dynamic environments, the queuing delay and the contention delay at the intermediate nodes are not taken into account for route selection. Thus, a minimum hop path may sometimes incur a higher end-to-end delay than some alternate paths. Moreover, routing protocols based on minimum number of hops cannot fairly distribute the routing load among mobile hosts. An unbalanced distribution of traffic may lead to higher packet dropping rate and faster battery power depletion on certain mobile nodes.

Several approaches, which consider the traffic load of a path as the main route selection criteria [4-6], have recently been proposed. Simulation results have shown that these loadbased routing protocols give a lower end-to-end delay when compared with minimum hop methods, e.g., the Ad hoc Ondemand Distance Vector (AODV) routing protocol.

In this paper, we propose a Delay-based Load-Aware Ondemand Routing (D-LAOR) scheme that utilizes both the estimated total path delay and the hop count as the route selection criterion. Since the overhead of redundant routing information can have serious impact on the overall performance of MANET [7], our proposed D-LAOR also has a mechanism in new route selection to avoid a congested node by selectively dropping the Route REQuest (RREQ) packets. Simulation results show that our proposed D-LAOR scheme increases packet delivery fraction and decreases end-to-end delay by more than $10 \%$ in a moderate network scenario when compared with the original AODV and other LAOR protocols.

The rest of this paper is organized as follows. In Section II, we describe three load-based routing mechanisms proposed in the literature and review the AODV routing protocol. In Section III, we describe the details of our proposed D-LAOR mechanism. Performance comparisons between our proposed scheme and other routing mechanisms are presented in Section IV. Finally, conclusions are given in Section V.

\section{RELATED WORK}

In this section, we summarize the load-based routing schemes proposed in the literature. These schemes utilize the load information as the path selection metric for routing in 


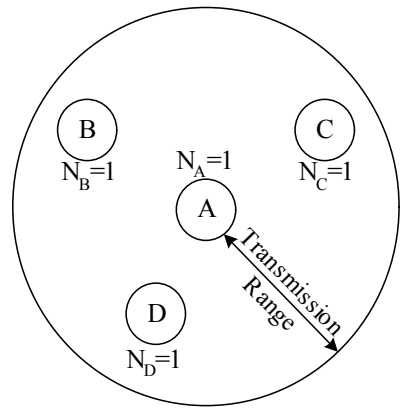

(a) Node A with three neighbors

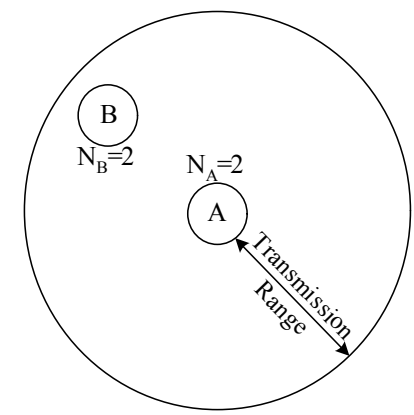

(b) Node A with one neighbor
Figure 1. Different delay with the same metric value.

MANETs. We also review the AODV routing protocol, which will be used for performance comparisons in Section IV.

\section{A. Load-Based Routing Protocols for MANETs}

In [4], Lee and Gerla proposed a Dynamic Load-Aware Routing (DLAR) protocol. They define the network load of a mobile node as the number of packets in its interface queue.

In [5], Hassanein and Zhou proposed a Load-Balanced Ad hoc Routing (LBAR) protocol. The network load in a node is defined as the total number of routes passing through the node and its neighbors.

In [6], Wu and Harms proposed a Load-Sensitive Routing (LSR) protocol. In LSR, the network load in a node is defined as the summation of the number of packets being queued in the interface of the mobile host and its neighboring hosts. Even though the load metric of LSR is more accurate than those of DLAR or LBAR, it does not consider the effect of access contentions in the MAC layer (e.g., IEEE 802.11). Therefore, LSR may regard different situations that may result in different access delays as the same in terms of traffic load metric. For example, node $A$ has different number of neighbors in Figures 1 (a) and (b). We define the number of packets being queued in the interface of the mobile host $k$ as $N_{k}$. Since the traffic load in LSR is the summation of the number of packets being queued in the interface of the mobile host and its neighboring hosts, the total traffic load of node $A$ in Figures 1(a) and (b) will be equal to four. However, the contention delay for node $A$ in Figure 1(a) is potentially higher than that in Figure 1(b).

In consideration of this contention delay, Sheu and Chen proposed a Delay-Oriented Shortest Path Routing (DOSPR) protocol [8] and analyzed the medium access delay of a mobile node in IEEE 802.11 wireless network.

The above observations motivate us to develop a new load aware on-demand routing protocol based on the estimation of the delay and the hop count of a path.

\section{B. Ad-Hoc On-Demand Distance Vector (AODV)}

The AODV protocol [9] is one of the routing protocols currently being studied within the IETF MANET working group. AODV is an on-demand variation of the distance vector routing protocols. When a source node desires to send a message to a certain destination node to which it does not have a valid route, it initiates a route discovery process. The source node broadcasts a RREQ packet to its neighbors, which then forward the request to their neighbors, and so on, until either the destination or an intermediate node with a route to the destination in its routing table is reached. During the process of forwarding the RREQ, an intermediate node record in its routing tables the address of the neighbor (precursor list) from which the first copy of the broadcast packet is received, thereby establishing a reverse path. Any additional copies of the same RREQ received later are discarded. Once the RREQ reaches the destination or an intermediate node with a route, the respective node responds by unicasting a Route REPly (RREP) packet back to the neighbor from which it first received the RREQ, which relays the RREP backward via the precursor nodes to the source node.

Routes are maintained as follows: when a source node moves, it has to re-initiate the route discovery protocol to find a new route to the destination. On the other hand, when an intermediate node along the route moves, its upstream neighbor will notice the movement and propagate a Route ERRor (RERR) packet to each of its active upstream neighbors. These nodes in turn propagate this RERR packet to their upstream neighbors, and so on until the source node is reached. The source node may then choose to re-initiate the route discovery for that destination if a route is still desired.

\section{DELAY-BASED LOAD-AWARE ON-DEMAND ROUTING (D-LAOR)}

In this section, we introduce the concept and operation of D-LAOR and present the rules for route selection and maintenance.

\section{A. Local Delay Measurement}

We assume that each mobile node uses the MAC protocol specified by IEEE 802.11 Distributed Coordination Function (DCF) [10] and can monitor the MAC layer interface queue.

A timestamp is placed on each packet header when the mobile node receives the packet. We use $a_{i}$ and $d_{i}$ to denote the arrival and successful transmission time of the $i^{\text {th }}$ packet, respectively. After the $i^{\text {th }}$ packet's successful transmission at a node $k$, the estimated average total node delay $q_{i}^{k}$ at node $k$ is computed as follows:

$$
q_{i}^{k}=(1-\beta) q_{i-1}^{k}+\beta\left(d_{i}-a_{i}\right)
$$

for $i>1$ and $0 \leq \beta \leq 1$.

The estimated average total delay includes queuing, contention, and transmission delays. We assume that the propagation delay is negligible. We define $D_{P}$ as the total path delay of a path $P$ from node 1 to $n . D_{P}$ is represented as follows:

$$
D_{P}=\sum_{k=1}^{n} Q_{k}
$$


where $Q_{k}$ denotes the estimated total node delay $q_{i}^{k}$ of node $k$ at the time of route discovery.

\section{B. Route Discovery and Maintenance}

Our proposed Delay based Load Aware On-demand Routing (D-LAOR) protocol is an extension of the AODV. We begin by describing the differences between our proposed D-LAOR and the original AODV:

1) D-LAOR allows the intermediate nodes to relay duplicate RREQ packets if the new path $\left(P^{\prime}\right)$ to the source of RREQ is shorter than the previous path $(P)$ in hop count, and $D_{P}$, is smaller than $D_{P}$ (i.e., $D_{P},<D_{P}$ ).

2) Each node updates the route entry only when the newly acquired path $\left(P^{\prime}\right)$ is shorter than the previous path $(P)$ in hop count, and $D_{P}$, is smaller than $D_{P}$ (i.e., $D_{P},<D_{P}$ ).

3) D-LAOR does not allow the intermediate nodes to generate a RREP packet to the source node to avoid the problem with stale path delay information.

When a source node does not have a valid route to a destination, it initiates a route discovery process. The source node broadcasts a RREQ packet to its neighbors, which then update the total path delay and forward this RREQ packet to their neighbors, and so on, until the destination is reached.

The RREQ packet carries the source and destination addresses, the sequence number, hop-count, and the total path delay $D_{P}$ of a path $P$, which the RREQ packet has traversed. During the process of forwarding this RREQ packet, intermediate nodes record in their routing tables the total path delay $D_{P}$ of the path $P$. Any duplicate RREQ packets received later will be relayed if the new path $P$ ' has a smaller hop count and a smaller path delay than the previous path (i.e., $D_{P},<D_{P}$ ).

Once the first RREQ packet has arrived at the destination, the destination node responds by unicasting a RREP packet back to the neighbor from which it received the corresponding RREQ packet. If the duplicate RREQ packet has a smaller total path delay and hop count than the previous one, the destination sends a RREP packet again to the source node to change the route immediately. D-LAOR does not allow any intermediate node to generate a RREP packet instead of the destination node because the intermediate node's record of the path delay to the destination may not be accurate.

When an established path is broken due to node mobility, a RERR packet is sent to the source node. The source node reinitiates the route discovery process as described above.

The example in Figure 2 shows the advantage of relaying duplicate RREQ packets at a mobile node. Source node $S$ broadcasts a RREQ packet to the network to find a route to destination node $D$. Assuming that there is a contention between node $A$ and its neighbors, the first RREQ packet can arrive through the path $S-B-C-D$ instead of the shortest path $S$ $A-D$. Note that even if the estimated average total node delay $Q_{A}$ is 1 in node $A$, instantaneous delay can be larger or smaller than 1 because of the dynamic nature of MANET. As shown in Figure 2(a), since the original AODV does not allow any intermediate nodes or the destination node to process a duplicate RREQ packet, node $D$ will choose the path $S-B-C-D$
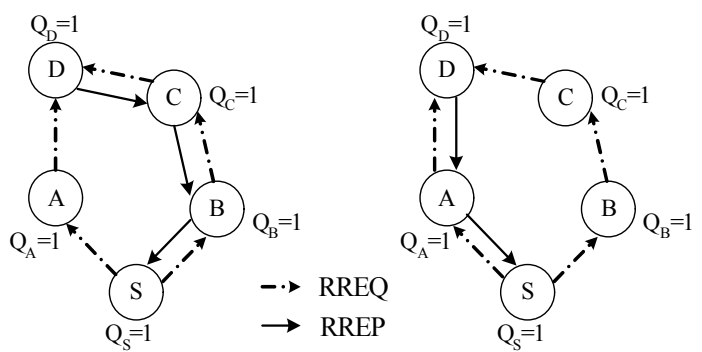

(a) No Duplicate RREQ (AODV)

(b) Duplicate RREQ (D-LAOR)

Figure 2. The effect of relaying duplicate RREQs.

as an optimal route to node $S$ in Figure 2(a). However, in DLAOR (Figure 2(b)), destination node $D$ will send another RREP packet via path $S-A-D$ since this path has a smaller total path delay than the path $S-B-C-D$, and has a smaller hop count.

Moreover, our proposed D-LAOR can route around a congested node and thus can reduce the control overhead. This is achieved by dropping the RREQ packets at congested nodes, which prevents the congested node from becoming an intermediate node of a path. D-LAOR determines the congested node by comparing the estimated total node delay and the number of packets being queued in the interface queue of two serial nodes in a RREQ packet-forwarding path. DLAOR drops a RREQ packet only when the following two conditions are satisfied simultaneously:

1) The estimated total node delay of a node $A$ is greater than that of previous node $B$.

2) The number of packets being queued at the interface queue of a node $A$ is more than $80 \%$ of its buffer size.

\section{Simulation Model ANd Evaluations}

\section{A. Simulation Environments}

We consider a network topology with 50 nodes randomly placed over a $1500 \times 300 \mathrm{~m}^{2}$ flat-grid. These nodes correspond to the mobile nodes. For the traffic model, we consider constant bit rate (CBR) data sources each sending packets at a fixed rate of 4 packets/sec. The data packet size is 512 bytes.

The random waypoint model used in [3] is adopted for the mobility model. In this mobility model, a node randomly selects a destination. Once the destination is reached, another random destination is targeted after some fixed pause time. Each simulation run takes 300 simulated seconds. For fair comparisons, identical mobility and traffic scenarios are applied to all routing protocols.

The physical radio characteristics of each mobile node's radio interface are chosen to approximate the Lucent WaveLAN [11], operating as a shared-media radio with a nominal bit rate of $2 \mathrm{Mbits} / \mathrm{sec}$ and a nominal radio range of $250 \mathrm{~m}$. For the MAC layer, the IEEE 802.11 DCF is used. Our propagation model combines both a free space propagation model and a two-ray ground reflection model. We use the same configuration parameters as those of ns2 [12] 
Table 1: Simulation parameters.

\begin{tabular}{|l|l||l|l|}
\hline $\begin{array}{l}\text { Transmission } \\
\text { range }\end{array}$ & $250 \mathrm{~m}$ & $\begin{array}{l}\text { Topology } \\
\text { size }\end{array}$ & $1500 \times 300 \mathrm{~m}^{2}$ \\
\hline $\begin{array}{l}\text { Bandwidth } \\
\text { of radio } \\
\text { interface }\end{array}$ & $2 \mathrm{Mbit} / \mathrm{sec}$ & Traffic type & CBR \\
\hline $\begin{array}{l}\text { Simulation } \\
\text { time }\end{array}$ & $300 \mathrm{sec}$ & Packet rate & 4 packets $/ \mathrm{sec}$ \\
\hline $\begin{array}{l}\text { Number of } \\
\text { nodes }\end{array}$ & 50 & Packet size & 512 Bytes \\
\hline $\begin{array}{l}\text { Number of } \\
\text { source nodes }\end{array}$ & $\begin{array}{l}20,25,30, \\
35,40\end{array}$ & $\begin{array}{l}\text { Max. mobile } \\
\text { speed }(\mathrm{m} / \mathrm{s})\end{array}$ & $5,10,15,20$ \\
\hline
\end{tabular}

Table 2: Route selection metrics.

\begin{tabular}{|l|l|}
\hline Routing protocol & Route selection metric \\
\hline \hline AODV & Hop-count \\
\hline D-LAOR with $\beta=\mathrm{X}$ & $\begin{array}{l}\text { Estimated total path delay with } \beta=\mathrm{X} \\
\text { in eqn. (1) and the hop-count }\end{array}$ \\
\hline F-LAOR & $\begin{array}{l}\text { Number of flows passing the node } \\
\text { and its neighbors of the path [5] and } \\
\text { the hop-count }\end{array}$ \\
\hline P-LAOR & $\begin{array}{l}\text { Number of packets being queued in } \\
\text { the interface of the node and its } \\
\text { neighbors of the path [6] and the } \\
\text { hop-count }\end{array}$ \\
\hline
\end{tabular}

version b8a. Table 1 provides a summary of the simulation parameters.

We compare three performance metrics for evaluations:

1) Packet delivery fraction: The measured ratio of the number of data packets delivered to the destinations to the number of packets generated by all traffic sources.

2) Average end-to-end delay of transferred data packets: This includes all possible delays caused by buffering during route discovery, queuing at the interface-queue, contention and retransmission delays at the MAC layer, and propagation and transfer times.

3) Routing overhead: The number of routing control packet transmissions per data packet delivered at the destination. Note that each hop of a routing control packet is counted as one packet transmission.

\section{B. Simulation Results and Evaluations}

Table 2 shows the comparison of route selection metrics of different routing protocols used in our simulations. To evaluate the effectiveness of our metric, we have implement two different route selection metrics, based on the ones used in the LBAR [5] and LSR protocols [6], but applied to AODV routing protocol, and refer to them as Flow-based LAOR (FLAOR) and Packet-based LAOR (P-LAOR), respectively, according to the implemented path selection metrics (see Table 2).

Figure 3 shows the packet delivery fraction, average end-toend delay, and routing overhead as a function of the speed of the mobiles. Results in Figure 3(a) shows that the D-LAOR (a) Delivery fraction vs. mobility

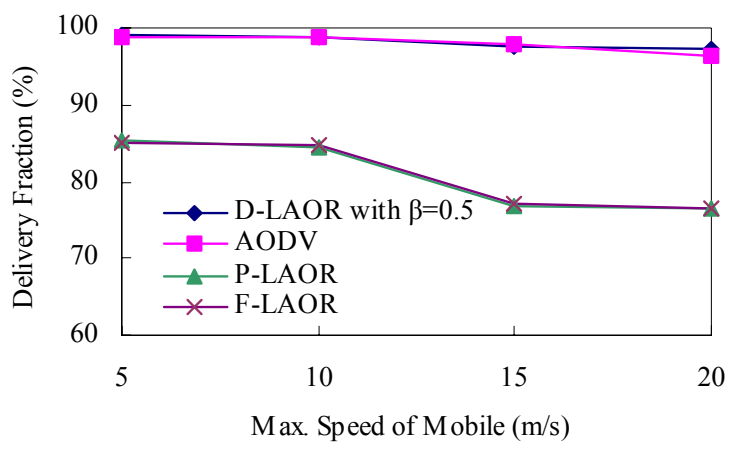

(b) End-to-end delay vs. mobility

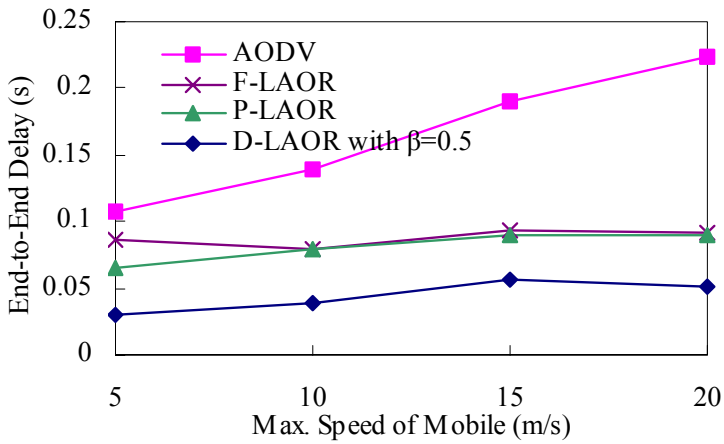

(c) Routing overhead vs. mobility

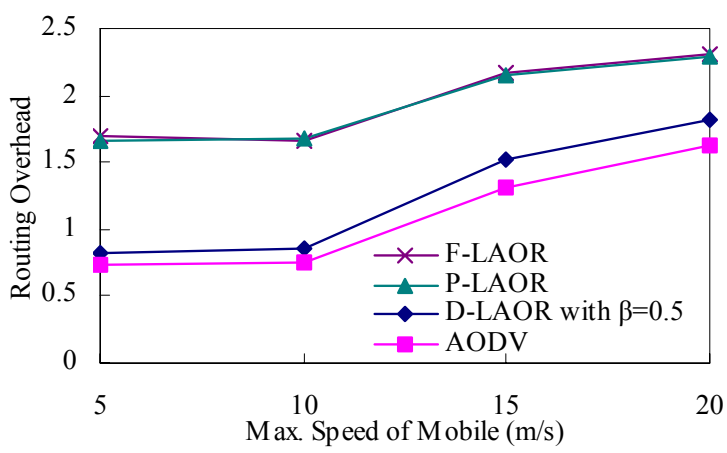

Figure 3. Performance comparison between LAORs and AODV with different speed of mobiles (number of CBR sources $=\mathbf{2 0}$ ).

and the original AODV maintain a higher packet delivery fraction than other two protocols. This is due to the fact that in both P-LAOR and F-LAOR, every node needs to send HELLO packets to its local neighbors periodically to update its neighbor's load information. Results in Figure 3(b) indicate that LAOR protocols have lower average end-to-end delay when compared with AODV. Results in Figure 3(c) show that AODV incurs a lower routing control overhead than LAOR protocols. This is because LAOR allows relaying the duplicate RREQ packets to detect a better path. The increase of the routing control overhead is due to the increase of the number of RREQ packets in D-LAOR, and due to the RREQ 
(a) Delivery fraction vs. number of sources

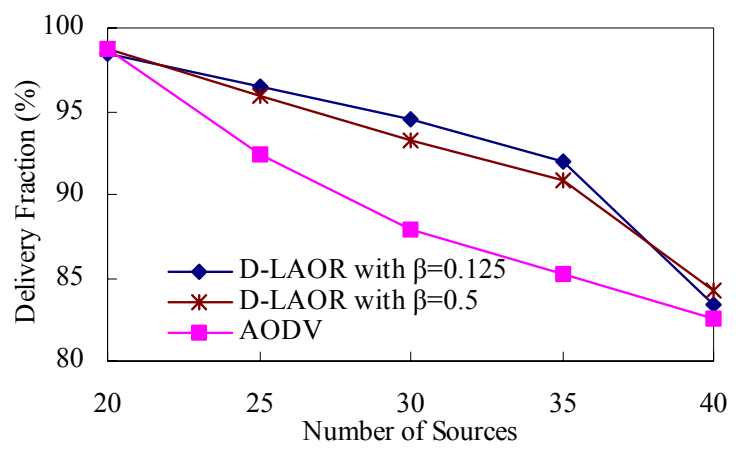

(b) End-to-end delay vs. number of sources

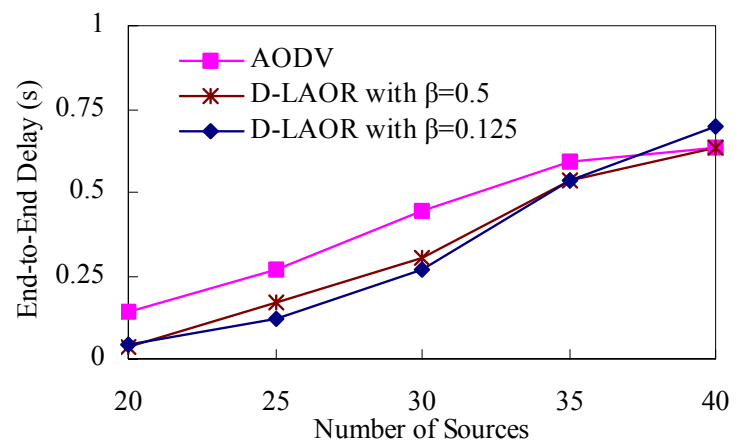

(c) Routing overhead vs. number of sources

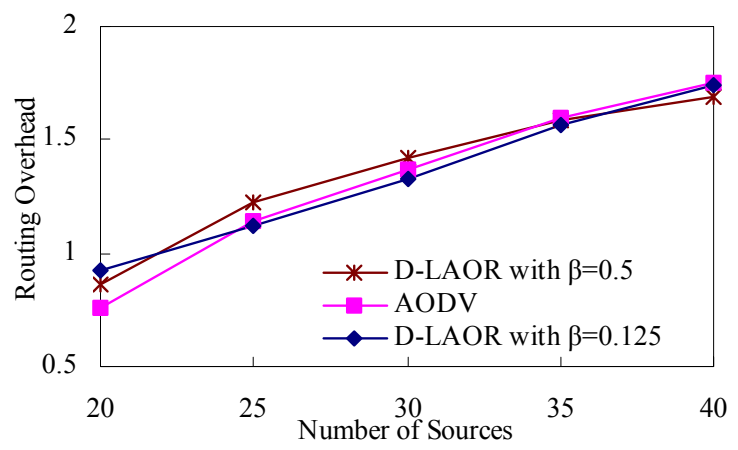

Figure 4. Performance comparison between D-LAORs and AODV with different number of CBR sources (speed of mobile $=10 \mathrm{~m} / \mathrm{s}$ ).

and HELLO packets in case of P-LAOR and F-LAOR protocols.

Figure 4 shows the packet delivery fraction, average end-toend delay, and routing overhead as a function of the number of CBR sources. Results in Figure 4(a) indicate that when the number of source nodes increases up to 30 , the difference of the packet delivery fraction between D-LAORs and AODV becomes significant. However, as the number of sources increases to 40, the performance improvement is not remarkable. This result shows that when traffic load is high, most routes are congested and the effectiveness of LAOR protocols is not significant. Figure 4(b) shows that average end-to-end delay increases as the number of sources increases.
Result in Figure 4(c) indicates that the routing overhead increases as the number of sources increases. However, these results show that the amount of routing overhead incurred between AODV and D-LAOR is similar. It indicates that the relaying of duplicate RREQ packets is compensated by dropping RREQ packets to avoid congested nodes.

\section{CONCLuSions}

In this paper, we have presented the delay-based load-aware on-demand routing scheme, D-LAOR for MANETs. We have compared the performance of different LAORs with AODV under different mobility and traffic scenarios. Over the range of mobile speeds and number of CBR sources considered in the simulations, our proposed D-LAOR outperforms not only AODV but also P-LAOR and F-LAOR protocols, by delivering more data packets to the destination or decreasing the end-to-end delay of packets delivered or both. Future work includes implementing the delay-based route selection scheme on other on-demand routing protocols.

\section{ACKNOWLEDGMENTS}

This work was supported by a University of British Columbia Graduate Fellowship, and by the Canadian Natural Sciences and Engineering Research Council under grant OGP0044286.

\section{REFERENCES}

[1] J. Macker and S. Corson, "Mobile Ad hoc Networks (MANET): Routing Protocol Performance Issues and Evaluation Considerations," IETF RFC 2501, Jan. 1999.

[2] X. Hong, K. Xu, and M. Gerla, "Scalable Routing Protocols for Mobile Ad Hoc Networks," IEEE Network, July/Aug. 2002.

[3] J. Broch, D.A. Maltz, D.B. Johnson, Y. Hu and J. Jetcheva, "A Performance Comparison of Multi-hop Wireless Ad Hoc Network Routing Protocols," in Proc. ACM MOBICOM'98, Dallas, Texas, Oct. 1998.

[4] S. -J. Lee and M. Gerla, "Dynamic Load-Aware Routing in Ad Hoc Networks," in Proc. IEEE ICC'01, Helsinki, Finland, June 2001.

[5] H. Hassanein and A. Zhou, "Routing with Load Balancing in Wireless Ad Hoc Networks," in Proc. ACM MSWiM, Rome, Itary, July 2001.

[6] K. Wu and J. Harms, "Load-Sensitive Routing for Mobile Ad Hoc Networks," in Proc. IEEE ICCCN'01, Scottsdale, AZ, Oct. 2001.

[7] Y. Yi, M. Gerla, and T.J. Kwon, "The Selective Intermediate Nodes Scheme for Ad Hoc On-Demand Routing Protocols," in Proc. IEEE ICC'02, New York City, New York, April/May 2002.

[8] S-T. Sheu and J. Chen, "A Novel Delay-Oriented Shortest Path Routing Protocol for Mobile Ad Hoc Networks," in Proc. IEEE ICC'01, Helsinki, Finland, June 2001.

[9] C. E. Perkins, E. M. Royer and S. R. Das, "Ad hoc On-Demand Distance Vector (AODV) Routing," Nov. 2002, IETF Internet Draft (work in progress).

[10] Wireless LAN Medium Access Control (MAC) and Physical Layer (PHY) specifications, ISO/IEC 8802-11; ANSI/IEEE Std 802.11, Aug. 1999.

[11] B. Tech "Development of WaveLAN, an ISM Band Wireless LAN," AT\&T Technical Journal, July/Aug. 1993.

[12] The Network Simulator - NS-2 notes and documentation and source code. Available from http://www.isi.edu/nsnam/ns/. 